\title{
The Relationships between Leptin, Genotype, and Chinese Medicine Body Constitution for Obesity
}

\author{
Hsiang-I. Hou $\mathbb{D}^{1}{ }^{1}$ Hsing-Yu Chen $\mathbb{D}^{1,2,3}$ Jang-Jih Lu $\mathbb{D}^{4,5,6}$ Shih-Cheng Chang $\mathbb{D}^{4,5}$ \\ Hsueh-Yu Li $\mathbb{D},,^{7,8}$ Kun-Hao Jiang $\mathbb{D},{ }^{1,3}$ and Jiun-Liang Chen $\mathbb{D}^{1,3}$ \\ ${ }^{1}$ Division of Chinese Internal Medicine, Center for Traditional Chinese Medicine, Chang Gung Memorial Hospital, \\ Taoyuan, Taiwan \\ ${ }^{2}$ Graduate Institute of Clinical Medical Sciences, College of Medicine, Chang Gung University, Taoyuan, Taiwan \\ ${ }^{3}$ School of Traditional Chinese Medicine, College of Medicine, Chang Gung University, Taoyuan, Taiwan \\ ${ }^{4}$ Department of Laboratory Medicine, Chang Gung Memorial Hospital, Taoyuan, Taiwan \\ ${ }^{5}$ Department of Medical Biotechnology and Laboratory Science, College of Medicine, Chang Gung University, Taoyuan, Taiwan \\ ${ }^{6}$ Department of Medicine, College of Medicine, Chang Gung University, Taoyuan, Taiwan \\ ${ }^{7}$ Department of Otolaryngology, Chang Gung Memorial Hospital, Gueishan County, Taoyuan, Taiwan \\ ${ }^{8}$ Department of Otolaryngology-Head and Neck Surgery, Sleep Center, Chang Gung Memorial Hospital, Taoyuan, Taiwan
}

Correspondence should be addressed to Jiun-Liang Chen; a12015@adm.cgmh.org.tw

Received 2 February 2021; Revised 6 March 2021; Accepted 30 April 2021; Published 8 May 2021

Academic Editor: Yong-Bo Xue

Copyright (C) 2021 Hsiang-I. Hou et al. This is an open access article distributed under the Creative Commons Attribution License, which permits unrestricted use, distribution, and reproduction in any medium, provided the original work is properly cited.

\begin{abstract}
Background and Purpose. Obesity is a widespread and increasingly severe problem worldwide. Several single nucleotide polymorphisms (SNPs) associated with obesity have been identified, reflecting metabolic disorders between the environment, diet, and body constitution. Traditional Chinese medicine body constitution (TCMBC) plays a central role in the classification of human body constitution from the viewpoint of traditional Chinese medicine (TCM). This study aims at exploring the relations between TCMBC and obesity-related biochemistry profiles and SNPs. Methods. The adults with body mass index (BMI) more than $27 \mathrm{~kg} / \mathrm{m}^{2}$ were enrolled in the study. General personal information, physical condition, TCMBC, biochemical, and SNPs were collected for eligible subjects. The body constitution questionnaire (BCQ) was used to evaluate the relationships between TCMBC tendency, biochemical values, and obesity-related SNPs. Results. Obesity patients tended to have a yin deficiency constitution (YinDC) $(n=33,66.0 \%)$; however, TCMBC in combination is not uncommon (30 subjects with more than two TCMBC in combination). For biochemical profiles, leptin was higher among patients with yang deficiency constitution (YangDC) (YangDC versus non-YangDC: $29.7 \pm 24.8$ versus $15.9 \pm 9.9, P=0.020$ ) and YinDC (YinDC versus non-YinDC: $28.8 \pm 23.5$ versus $14.4 \pm 9.6$, $P=0.020)$. The leptin level was highest among YangDC subjects. Higher leptin was found among subjects with three-combined TCMBC than balanced TCMBC subjects who were not inclined to any of three TCMBC. For obesity-related SNPs, the adrenergic receptor beta-3 (ADRB3) gene tended to be high expression among YangDC (YangDC versus non-YangDC: 89.7\% versus 71.4\%, $P=0.091$ ) and uncoupling protein 1 (UCP1) tended to be high expression among phlegm-stasis constitution (PSC) (PSC versus non-PSC: $37.9 \%$ versus $9.5 \%, P=0.052)$. Conclusions. The relationships between TCMBC, leptin, and SNPs present alternative viewpoints about TCMBC and could be used as a guide to treat obese patients.
\end{abstract}

\section{Introduction}

Obesity is a complex, chronic medical condition with a significant negative impact on human health [1]. Since the 21 st century, the global obesity population has snowballed. Men's prevalence rate has risen from $4.8 \%$ to $9.8 \%$, women have risen from $7.9 \%$ to $13.8 \%$, and the global prevalence rate is $34.3 \%$ [2]. Obesity currently affects 78.6 million people $(33 \%)$ in the United States and is expected to increase to over $50 \%$ of the population by 2030 [3]. In Taiwan, the prevalence of overweight is $30.5 \%$ for men and $21.3 \%$ for women, and the prevalence of obesity is $19.2 \%$ for men and 
$13.4 \%$ for women [4]. Obesity has become a public health burden with a significant and profound impact on morbidity, mortality, and healthcare costs [1]. Patients with obesity are at increased risk of morbidity from dyslipidemia, type 2 diabetes, hypertension, coronary heart disease, stroke, gallbladder disease, respiratory problems, sleep apnea, osteoarthritis, and some cancers [5].

There are many reasons for obesity, which can be divided into congenital and acquired. In addition to the acquired causes such as dietary habits, physical activities, and living habits, the heredity of the genetic constitution is also valued by scholars [6]. The heritability of body mass index (BMI) has been estimated to be $40-70 \%$, and genome-wide association studies for adiposity traits have identified more than 300 single nucleotide polymorphisms (SNPs) [7, 8]. For example, the adrenergic receptor beta-2 (ADRB2), adrenergic receptor beta-3 (ADRB3), guanine nucleotide-binding protein (GNB3), uncoupling protein 1 (UCP1), and fat mass- and obesity-associated (FTO) genes are all reported to have associations with obesity. ADRB2 rs1042714 SNP may contribute to the risk of obesity and predict obesity-related metabolic traits such as BMI, triglyceride, and systolic blood pressure in Taiwanese subjects [9]. Trp64Arg (rs4994) polymorphism in the ADRB3 gene was significantly higher in overweight and obese subjects than normal weight subjects [10]. Studies regarding the role of GNB3 rs5443 polymorphism in the development of obesity and its related comorbidities are contradictory [11]. The rs 1800592 of the UCP1 gene is associated with obesity in general and in the moderate obese group in particular. The associated UCP1 polymorphisms in the moderate obese group may regulate the impaired energy metabolism, which plays a significant role in the initial stages of obesity [12]. The FTO gene is highly expressed in the hypothalamus, visceral fat, and liver. It contributes to an inflammatory state, food intake, and appetite $[13,14]$. A study on impaired glucose regulation patients found that leptin serum levels increased in subjects with the yang deficiency constitution and phlegm-stasis constitution, but not obese patients [15].

For traditional Chinese medicine (TCM) doctors, the constitution is one of the most important clinical references to treating diseases. The constitution is broadly defined as the fundamental components that constitute a human being and includes the total expression of physiological, psychological, and pathological traits that characterize a person's health [16]. It is a relatively stable characteristic of an organism and is affected by both nature and nurture. Traditional Chinese medicine body constitution (TCMBC) studies the overall physical condition affected by genetic and acquired factors. Body composition lays the foundation for the diagnosis, prevention, and treatment of diseases. Different constitution types make individuals susceptible to different diseases. Examining the individual's unique body constitution can promote effective health management and greatly benefit personalized medicine [16]. However, the relations between TCMBC, SNPs, and obesity-related biochemical profiles are less studied.

The present study aimed to investigate the association between TCMBC and obesity-related polymorphisms in obese patients. Our results could help to identify abnormal constitutions with a high risk for obesity and help clinicians focus on preventive strategies more effectively on these patients.

\section{Materials and Methods}

2.1. The Study Protocol and Enrollment. We prospectively conducted this study from January 2018 to December 2019 at the Department of Traditional Chinese Medicine of Chang Gung Memorial Hospital (Taoyuan and Taipei, Taiwan). The age, sex, BMI, waist-hip ratio, waist-height ratio, lifestyles, obesity-related genotyping and biochemical profiles, and comorbidities were collected, and TCMBC was measured. This study was approved by the Institutional Review Board (IRB) of Chang Gung Memorial Foundation (IRB no.: 201701723B0). All the study participants gave informed consent and signed a therapeutic partnership agreement. The inclusion criteria were the following: (1) BMI $\geq 27 \mathrm{~kg} /$ $\mathrm{m}^{2}$; (2) age $\geq 20$ years; (3) no recognition disabilities; and (4) willing to sign informed consent and tolerance to blood sampling and questionnaire filling. The exclusion criteria were the following: (1) pregnant women or planning to conceive; (2) a history of endocrine disorders other than diabetes mellitus; (3) history of psychiatric disease and not compliance to blood sampling or questionnaire filling; (4) impaired activity of daily living; and (5) record of other ongoing clinical trials.

\subsection{Body Constitution Questionnaire for TCMBC Assessment.} Professor Yi-Chang Su kindly approved the use of the body constitution questionnaire (BCQ), Ph.D., School of Chinese Medicine, Chinese Medical University (Taichung, Taiwan), and BCQ has been widely used for TCM constitution assessments $[17,18]$. All participants' TCMBC were assessed using BCQ by a TCM doctor, and the tendency to three types of TCMBC was calculated at the end of this study. The questionnaire consists of 44 items of a 5-point Likert-type response scale (from 1 (never happened) to 5 (always happens)) with a total score ranging from 44 to 220. BCQ was designed to detect the TCMBC tendency: Yang deficiency constitution (YangDC), yin deficiency constitution (YinDC), and phlegm-stasis constitution (PSC) separately. For YangDC (total score ranging from 19 to 95, 19 items), a score exceeding 31 indicated YangDC [19]; for YinDC (total score ranging from 19 to 95, 19 items), a score exceeding 30 indicated YinDC [20]; and for PSC (total score ranging from 16 to 80,16 items), a score exceeding 27 indicated PSC [21]. A higher score implies a more significant deviation of the body constitution. Although these three TCMBC could be seen as three different, some items belonging to these three scales overlapped each other, so the body constitution classification may also be overlapped. Subjects who did not reach the threshold scores of all three imbalanced body constitutions were regarded as a balanced constitution where the lower the score, the healthier the subject. In previous studies, Cronbach's confidence $\alpha$ of each constitution's subscale ranged from 0.55 to 0.88 , with the intraclass correlation coefficients exceeding $0.7[17,18,21]$. 
2.3. Determination of Clinical and Biochemical Profiles. The following variables were recorded for each obesity patient: gender, age, BMI, fasting sugar, hemoglobin A1C (HbAlc), estimated average glucose(eAG), high-density lipoprotein-cholesterol (HDL-c), very low-density lipoprotein (VLDL), low-density lipoprotein-cholesterol (LDL-c), total cholesterol, triglyceride (TG) leptin, insulin, and adiponectin. The homeostatic model assessment for insulin resistance (HOMA-IR) was used to represent insulin resistance.

2.4. Determination of SNP Selection and Genotyping. The ADRB2 gene is a vital lipolysis receptor on human fat cells. Arg16Gly (rs1042713) polymorphism of ADRB2 was significantly associated with obesity in female adolescents in Taiwan, and Gln27Glu (rs1042714) polymorphism of ADRB2 may contribute to the risk of obesity and predict obesity-related metabolic traits such as BMI, triglyceride, and systolic blood pressure in Taiwanese subjects $[9,22]$. In the ADRB2 gene, Gln27Glu polymorphism is widespread in obesity and associates with increased body fat and enlarged fat cells, and Arg16Gly polymorphism is above all associated with improved adipocyte ADRB2 function [23]. Women with the Gln27Glu polymorphism in the ADRB2 gene on a high intake of carbohydrates ( $>50 \%$ of daily calories intake) increase the risk of obesity [24]. Those with the ADRB2 gene are called starch obesity. The ADRB3 gene regulates the lipolysis of adipose tissue. ADRB3 polymorphism might be a possible determinant of insulin resistance in Taiwanese women [25]. Middle-aged adult Asians with the ADRB3 rs4994 minor alleles are at increased risk of type 2 diabetes [26]. For people with this genotype, the lipolysis of visceral adipose tissue is reduced, which leads to higher visceral fat content, and the weight and BMI value are easily increased [27]. Those with the ADRB3 gene are called visceral obesity. The GNB3 gene slows down cell fat metabolism, causing cells to accumulate fat quickly and cause obesity [28]. The GNB3 rs5443 polymorphism is significantly associated with greater visceral fat and higher serum lipids in Korean obese women [28]. GNB3 rs5443 SNP may predict higher obesity-related metabolic traits such as triglyceride and total cholesterol in nonobese Taiwanese subjects [29]. People with the GNB3 gene are called metabolism obesity. The UCP1 gene acts on the inner mitochondrial membrane of brown fat cells and can convert energy to generate heat [30]. A Japanese study showed that UCP1 showed significant associations with visceral fat area [31]. When carrying the UCP1 gene, BMI, waist circumference, and waist-to-hip ratio values are higher than normal genes, so fat is easy to accumulate in the hips and thighs and cause lower body obesity, also known as stubborn obesity [32]. The FTO gene affects the pathway of fat cells to produce heat, so that the fat type is transformed into white fat cells, and the fat storage is increased [33]. FTO rs9939609 SNP may be linked with the risk of obesity in Taiwanese subjects [34]. The FTO gene can regulate the secretion of ghrelin and affect appetite [35]. People with the FTO gene will significantly increase their food intake, increasing the risk of rising BMI, as known as appetite obesity.
2.5. Physical Examination. All subjects also had physical examination analyses in this study. Bodyweight and height were measured with subjects dressed in light clothing without socks and shoes. Weight was measured (in kilograms to one decimal place) with a digital balance scale. Height was measured (in meters to one decimal place) with an electronic height measuring instrument. Body mass index (BMI) was calculated in the way that bodyweight was divided by body height squared. Waist circumference (WC) and hip circumference ( $\mathrm{HC}$ ) were measured to the nearest $0.1 \mathrm{~cm}$ using a standard tape. Waist circumference (WC) was measured between the lowest rib and the superior border of the iliac crest. Hip circumference (HC) was measured as the maximum circumference around the buttocks. Systolic and diastolic blood pressure was measured in the seated position with and an appropriately sized cuff on the left arm.

2.6. Sample Collection, SNP Selection, and Genotyping. In this study, genomic DNA was extracted from $3 \mathrm{~mL}$ peripheral blood samples of 50 individuals using the QIAamp DNA Blood Mini Kit according to the protocol recommended by the manufacturer (Qiagen, Valencia, CA, USA). The quantity and quality were determined using a NanoDrop (Thermo Fisher Scientific, Waltham, MA, USA). The obesity-related loci selected in this study were associated with BMI or energy intake in previously published genome-wide and candidate gene association studies (Table 1). All six obesity-related loci were genotyped in our 60 cases using the Sequenom MassARRAY platform and the standard protocol recommended by the manufacturer (Sequenom, San Diego, CA, USA). DNA samples were genotyped using the Sequenom MassARRAY platform (Agena Bioscience, San Diego, CA, USA). Primers and multiplex reactions were designed using the Agena Bioscience Assay Designer software package (v.4.0). Data management and analysis were performed using Agena Bioscience Type 4.0 software.

2.7. Statistical Analysis. Demographic features, including physical and biochemical profiles, were presented either by the mean and standard deviation for continuous covariates or percentage for categorical data. To compare the differences within three different TCMBC groups, Student's $t$-test was used for continuous variables, and $\chi^{2}$ statistics were used for categorical data. All statistics were calculated by STATA (StataCorp. 2019. Stata Statistical Software: Release 16. College Station, TX:StataCorp LLC.), and the statistics $\leq 0.05$ were regarded as significant results.

\section{Results}

3.1. Subjects' Demographic Characteristics. A total of 50 obese subjects were assessed for eligibility from January 1 , 2018, to December 31, 2019. After a detailed explanation of the study protocol and a full period of consideration, all patients provided informed consent. Patient characteristics, such as demographic features, TCMBC, and comorbidities data, are presented in Table 2 . The study group included 13 $(26.0 \%)$ males and $37(74.0 \%)$ females with a median age of 
TABLE 1: The list of obesity-related SNPs checked in this study.

\begin{tabular}{|c|c|c|}
\hline Gene & Phenotype & Reference \\
\hline $\begin{array}{l}\text { ADRB2 } \\
\text { rs1042713rs1042714 }\end{array}$ & Starch obesity & $\begin{array}{l}\text { V. Large, L. Hellstrom, S. Reynisdottir et al. "Human beta-2 adrenoceptor gene } \\
\text { polymorphisms are highly frequent in obesity and associate with altered adipocyte beta-2 } \\
\text { adrenoceptor function," J Clin Invest, vol. 100, no. 12, pp. 3005-3013. } \\
\text { J.A. Martínez, M.S. Corbalán, A. Sánchez-Villegas, L. Forga, A. Marti, and M.A. Martínez- } \\
\text { González, "Obesity risk is associated with carbohydrate intake in women carrying the } \\
\text { Gln27Glu beta-2 adrenoceptor polymorphism," J Nutr, vol. 133, no. 8, pp. 2549-2554. }\end{array}$ \\
\hline ADRB3 rs4994 & Visceral obesity & $\begin{array}{l}\text { H. Kim-Motoyama, K. Yasuda, T. Yamaguchi et al., "A mutation of the beta-3 adrenergic } \\
\text { receptor is associated with visceral obesity but decreased serum triglyceride," Diabetologia, } \\
\text { vol. } 40 \text {, no. } 4 \text {, pp. } 469-472 .\end{array}$ \\
\hline GNB3 rs5443 & $\begin{array}{c}\text { Metabolism } \\
\text { obesity }\end{array}$ & $\begin{array}{l}\text { K.D. Ko, K.K. Kim, H.S. Suh, and I.C. Hwang, "Associations between the GNB3 C825 T } \\
\text { polymorphism and obesity-related metabolic risk factors in Korean obese women," J } \\
\text { Endocrinol Invest, vol. } 37 \text {, no. 11, pp. 1117-1120. }\end{array}$ \\
\hline UCP1 rs1800592 & Stubborn obesity & $\begin{array}{l}\text { R.A. Busiello, S. Savarese, and A. Lombardi, "Mitochondrial uncoupling proteins and energy } \\
\text { metabolism," Front Physiol, vol. 6, p. } 36 \text {. } \\
\text { M. Dhall, M.M. Chaturvedi, U. Rai, and S. Kapoor, "Sex-dependent effects of the UCP1 } \\
\text {-3826 A/G polymorphism on obesity and blood pressure," Ethn Dis, vol. 22, no. 2, pp. } \\
181-184 .\end{array}$ \\
\hline FTO rs9939609 & Energy intake & $\begin{array}{l}\text { E. Karra, O.G. O'Daly, A.I. Choudhury et al. "A link between FTO, ghrelin, and impaired } \\
\text { brain food-cue responsivity," J Clin Invest, vol. 123, no. 8, pp. 3539-3551. } \\
\text { M. Claussnitzer, S.N. Dankel, K.H. Kim et al., "FTO obesity variant circuitry and adipocyte } \\
\text { browning in Humans," N Engl J Med, vol. 373, no. 10, pp. 895-907. }\end{array}$ \\
\hline
\end{tabular}

37.0 years and an average BMI of $31.1 \mathrm{~kg} / \mathrm{m}^{2}$. Obesity patients tended to have YinDC $(n=33,66.0 \%)$, followed by YangDC $(n=29,58.0 \%)$ and PSC $(n=29,58.0 \%)$. Moreover, combined TCMBC was quite commonly seen in enrolled patients. More than half of enrolled subjects have combined TCMBC ( $44 \%$ of subjects had three-combined TCMBC, and $16 \%$ had two-combined TCMBC). Eleven subjects (22\% of all enrolled subjects) had balanced TCMBC. The most common comorbidities of patients in this experiment were allergic rhinitis $(n=10,20 \%)$ and hypertension $(n=8,16 \%)$.

3.2. The Differences in Demographic Features among 3 TCMBC Groups. In Table 3, all demographic features and comorbidities were similar among three TCMBC groups, except gender. We found that there are differences between YangDC (female versus male: $89.7 \%$ versus $10.3 \%$, $P=0.003$ ) and YinDC (female versus male: $87.9 \%$ versus $12.1 \%, P=0.002)$ in gender. In terms of food preferences, there is a statistical difference in the intake of icy food (PSC versus non-PSC: $34.5 \%$ versus $9.5 \%, P=0.041$ ) and deepfried food (PSC versus non-PSC: $31.0 \%$ versus $4.8 \%$, $P=0.022)$ in PSC. YinDC has a statistical difference in alcohol consumption (YinDC versus non-YinDC: $39.4 \%$ versus $70.6 \%, P=0.037$ ) and diabetes mellitus (YinDC versus non-YinDC: $0.0 \%$ versus $11.8 \%, P=0.044)$.

3.3. Leptin Associated with Different TCMBC Groups. In the biochemical test, as given in Table 4, leptin was found to have statistically significant differences in the types of constitutions of YangDC (YangDC versus non-YangDC: $29.7 \pm 24.8 \mathrm{ng} / \mathrm{mL}$ versus $15.9 \pm 9.9 \mathrm{ng} / \mathrm{mL}, P=0.020)$ and YinDC (YinDC versus non-YinDC: $28.8 \pm 23.5 \mathrm{ng} / \mathrm{mL}$ versus $14.4 \pm 9.6 \mathrm{ng} / \mathrm{mL}, P=0.020)$, and we found YangDC $(29.7 \pm 24.8 \mathrm{ng} / \mathrm{mL})$ has a higher leptin value than PSC
$(25.9 \pm 21.4 \mathrm{ng} / \mathrm{mL})$. Insulin has a statistically different trend in YinDC (YinDC versus non-YinDC: $13.0 \pm 5.3 \mathrm{uU} / \mathrm{mL}$ versus $10.4 \pm 4.4 \mathrm{uU} / \mathrm{mL}, P=0.088)$. On the other hand, blood sugar levels, lipid profiles, insulin resistance, and adiponectin were similar among different TCMBC groups.

3.4. The Associations between Obesity-Related Genotyping and $T C M B C$. Table 5 summarizes the genotyping results, and we found YangDC tended to associate with a higher mutation rate of the ADRB3 gene (proportion of high mutation rate $89.7 \%$ for YangDC and $71.4 \%$ for non-YangDC obese subjects, $P=0.091$ ). PSC tended to associate with the high mutation rate of the UCP1 gene (proportion of high mutation rate $37.9 \%$ for PSC and $9.5 \%$ for non-PSC obese subjects, $P=0.052$ ). Otherwise, FTO, GNB3, and ADRB2 had no differences among the three TCMBC groups.

3.5. The Comparison between Three-Combined TCMBC and Balanced Constitution. Tables 6 and 7 summarize all the study parameters and compare the different features between the three-combined and the balanced TCMBC. Overall, the tendency was similar to the findings in the comparisons within the three TCMBC. Among demographic features, subjects with three-combined TCMBC tended to be female, preferred deep-fried food, and had a diabetes mellitus history. Leptin level was markedly higher for 3-combined TCMBC subjects. For genomic profile, there were no differences between 3-combined and balanced TCMBC subjects.

\section{Discussion}

Our study found that leptin was related to YangDC and YinDC, and there was a trend between ADRB3 
TABLE 2: Baseline characteristics of enrolled obese patients (from January 1, 2018, to December 31, 2019, $n=50$ ).

\begin{tabular}{|c|c|}
\hline Parameters & Subject number (\%) \\
\hline \multicolumn{2}{|l|}{ Demographic features } \\
\hline \multicolumn{2}{|l|}{ Gender } \\
\hline Female & $37(74.0)$ \\
\hline Male & $13(26.0)$ \\
\hline Age (years), mean (SD) & $37.0(9.6)$ \\
\hline $\mathrm{BMI}$, mean (SD) & $31.1(3.2)$ \\
\hline Waist-hip ratio (WHR), mean (SD) & $0.9(0.1)$ \\
\hline Waist-height ratio (WheR), mean (SD) & $0.6(0.0)$ \\
\hline \multicolumn{2}{|l|}{ Appetite } \\
\hline Poor & $2(4.0)$ \\
\hline Fair & $43(86.0)$ \\
\hline Good & $5(10.0)$ \\
\hline \multicolumn{2}{|l|}{ Food preference } \\
\hline Icy food & $12(24.0)$ \\
\hline Deep-fried food & $10(20.0)$ \\
\hline Smoking & $3(6.0)$ \\
\hline Alcohol consumption & $25(50.0)$ \\
\hline Betel nuts chewing & $0(0)$ \\
\hline Exercise $\geq 30 \mathrm{mins} /$ week & $40(80.0)$ \\
\hline \multicolumn{2}{|l|}{ Comorbidities } \\
\hline Hyperlipidemia & $2(4.0)$ \\
\hline Hypertension & $8(16.0)$ \\
\hline Diabetes mellitus & $2(4.0)$ \\
\hline Stroke & $0(0)$ \\
\hline Myocardial infarction & $0(0)$ \\
\hline Allergic rhinitis & $10(20.0)$ \\
\hline Polycystic ovarian syndrome & $1(2.0)$ \\
\hline \multicolumn{2}{|c|}{ The tendency of TCM body constitution (TCMBC) } \\
\hline Yang deficiency constitution (YangDC) & $29(58.0)$ \\
\hline Yin deficiency constitution (YinDC) & $33(66.0)$ \\
\hline Phlegm-stasis constitution (PSC) & $29(58.0)$ \\
\hline \multicolumn{2}{|l|}{ TCMBC in combinations } \\
\hline 0 & $11(22.0)$ \\
\hline 1 & $9(18.0)$ \\
\hline 2 & $8(16.0)$ \\
\hline 3 & $22(44.0)$ \\
\hline
\end{tabular}

polymorphism rs4994 and YangDC, while there was also a similar correlation between UCP1 polymorphism rs1800592 and PSC. To the best of our knowledge, this is the first study addressing the correlations between constitution types and polymorphisms, which might help ameliorate the advances of precision TCM. TCMBC could indicate individuals' overall health status, and an unbalanced body constitution often represents deteriorated health status, a condition that Western medicine will diagnose as no disease. With objective biochemical and genomic profiles, TCMBC may help classify the obesity population in more detail as part of personalized medicine.

Most interestingly, we found that the leptin level was higher among YangDC and YinDC when compared to nonYangDC and non-YinDC subjects, respectively. The overall values of age, BMI, and high leptin were similar to other studies about the obesity population, and these facts proved the need for other classification systems. A study on the subSaharan African population showed that patients were about 50-55 years old, for males, the average value of BMI was 25.3 and leptin was 4.8 , and for females, the average value of BMI was 28 and leptin was 18.5 [36]. Another German study showed that the included patients were 33-36 years old, for males, the average value of BMI was 26.0 and leptin was 6.5, and for females, the average value of BMI was 26.3 and leptin was 20.5 [37]. For TCMBC, the leptin serum level was higher with YangDC and PSC among the impaired glucose regulation patients [15]. Although obesity may be associated with impaired glucose regulation, the constitution seemed to be changing with different physical conditions. Besides, we found that combined TCMBC was common among obese subjects, and the tendency of higher leptin was found among three-combined TCMBC subjects. These results reflected that the mechanisms and presentations were quite complicated, and therefore, the classification of TCMBC may not be inclined to just one type in the real world.

Leptin is an adipocyte-secreted hormone that circulates primarily at levels proportional to the amount of adipose tissue, signaling long-term energy storage, and secondarily at levels modified by acute changes in caloric intake [38]. Leptin generally regulates energy homeostasis, decreases energy intake, and increases energy expenditure [38]. Obesity is characterized by elevated leptin levels or hyperleptinemia and resistance to the anorectic and bodyweightreducing effects of leptin [39]. From the TCM's viewpoint, the body constitution means the physiological state is maintained by the dynamic combination of energy (yang) and materials (yin) in the body, just as the role of leptin in fat metabolism. The constitution theory serves as an essential foundation of clinical TCM practice and has been applied for more than 2,000 years to evaluate patients in states of subhealth, subdisease, or predisease [40]. YinDC refers to individuals' materials to perform or maintain body functions that have diminished [20]. YangDC refers to a diminishing energy level in the body's physiological functioning [19]. An imbalance between energy and material can cause both YangDC and YinDC to a considerable extent in the human body, leading to obesity, which in turn leads to higher leptin values for YangDC and YinDC subjects. Besides, obese patients with YangDC had higher leptin levels compared with the other groups. Duan et al. reported that the increased leptin level was significantly and positively correlated with BMI and waist circumference in obese women [41]. The higher leptin level increased the risk of metabolic syndrome in Taiwanese individuals [42]. YangDC is one important pathogenesis of obesity, which is a high-risk factor of metabolic syndrome and diabetes [43]. Hence, we consider that YangDC in obese patients might have a greater influence on the regulation of obesity-related hormone peptides.

Additionally, we found the most common TCMBC was YinDC, although the patient number of YinDC was close to the patient number of YangDC and PSC. Li et al. reported the significant correlations between yang deficiency and phlegm-stasis groups with overweight and obesity outcomes with a different TCMBC questionnaire [44]. A study on diagnosis in prediabetes people found that the predominant TCMBC for prediabetes people was yin deficiency [45]. A study showed that the yin deficiency type was significantly correlated with hypertension and diabetes mellitus [46]. Another study suggested that phlegm-stasis and yin 
TABLE 3: Comparisons of demographic features and comorbidities with three traditional Chinese medicine body constitutions (TCMBC).

\begin{tabular}{|c|c|c|c|c|c|c|c|c|c|}
\hline \multirow{2}{*}{ Parameters } & \multicolumn{3}{|c|}{ Yang deficiency (YangDC) } & \multicolumn{3}{|c|}{ Yin deficiency (YinDC) } & \multicolumn{3}{|c|}{ Phlegm-stasis (PSC) } \\
\hline & $(+) n=29$ & $(-) n=21$ & $P$ & $(+) n=33$ & $(-) n=17$ & $P$ & $(+) n=29$ & $(-) n=21$ & $P$ \\
\hline \multicolumn{10}{|l|}{ Demographic features } \\
\hline Gender & & & 0.003 & & & 0.002 & & & 0.097 \\
\hline Female & $26(89.7 \%)$ & $11(52.4 \%)$ & & $29(87.9 \%)$ & $8(47.1 \%)$ & & $24(82.8 \%)$ & $13(61.9 \%)$ & \\
\hline Male & $3(10.3 \%)$ & $10(47.6 \%)$ & & $4(12.1 \%)$ & $9(52.9 \%)$ & & $5(17.2 \%)$ & $8(38.1 \%)$ & \\
\hline Age (years), mean (SD) & $37.7(10.3)$ & $36.0(8.7)$ & 0.54 & $37.9(9.8)$ & $35.2(9.2)$ & 0.34 & $37.1(10.3)$ & $36.8(8.8)$ & 0.89 \\
\hline BMI, mean (SD) & $30.8(3.2)$ & $31.6(3.2)$ & 0.42 & $31.1(3.1)$ & $31.2(3.5)$ & 0.93 & $30.8(3.2)$ & $31.6(3.3)$ & 0.43 \\
\hline Waist-hip ratio (WHR), mean (SD) & $0.9(0.1)$ & $0.9(0.0)$ & 0.20 & $0.9(0.1)$ & $0.9(0.0)$ & 0.19 & $0.9(0.1)$ & $0.9(0.0)$ & 0.56 \\
\hline Waist-height ratio (WheR), mean (SD) & $0.6(0.1)$ & $0.6(0.0)$ & 0.67 & $0.6(0.0)$ & $0.6(0.0)$ & 0.35 & $0.6(0.1)$ & $0.6(0.0)$ & 0.24 \\
\hline Appetite & & & 0.10 & & & 0.57 & & & 0.35 \\
\hline Poor & $2(6.9 \%)$ & $0(0.0 \%)$ & & $2(6.1 \%)$ & $0(0.0 \%)$ & & $2(6.9 \%)$ & $0(0.0 \%)$ & \\
\hline Fair & $26(89.7 \%)$ & $17(81$. & & $28(84.8 \%)$ & $15(88$ & & $25(86.2 \%)$ & 18( & \\
\hline Good & $1(3.4 \%)$ & $4(19.0 \%)$ & & $3(9.1 \%)$ & $2(11.8 \%)$ & & $2(6.9 \%)$ & $3(14.3 \%)$ & \\
\hline \multicolumn{10}{|l|}{ Food preference } \\
\hline Icy food & $9(31.0 \%)$ & $3(14.3 \%)$ & 0.17 & $9(27.3 \%)$ & $3(17.6 \%)$ & 0.45 & $10(34.5 \%)$ & $2(9.5 \%)$ & 0.041 \\
\hline Deep-fr & $8(2$ & & 0.12 & $8(24$. & 2( & 0.30 & $9(31$. & 1 & 0.022 \\
\hline Smoking & $2(6.9 \%)$ & $1(4.8 \%)$ & 0.75 & $2(6.1 \%)$ & $1(5.9 \%)$ & 0.98 & $2(6.9 \%)$ & $1(4.8 \%)$ & 0.75 \\
\hline Alcohol con & $12(41.4 \%)$ & $13(61.9 \%)$ & 0.15 & $13(39.4 \%)$ & $12(70.6 \%)$ & 0.037 & $13(44.8 \%)$ & $12(57.1 \%)$ & 0.39 \\
\hline Exercise $\geq 30 \mathrm{mi}$ & $23(79.3 \%)$ & $17(81.0 \%)$ & 0.89 & $27(81.8 \%)$ & $13(76.5 \%)$ & 0.65 & $24(82.8 \%)$ & $16(76.2 \%)$ & 0.57 \\
\hline \multicolumn{10}{|c|}{ (2) } \\
\hline Hyperlipidemia & $1(3.4 \%)$ & $1(4.8 \%)$ & 0.82 & $2(6.1 \%)$ & $0(0.0 \%)$ & 0.30 & $1(3.4 \%)$ & $1(4.8 \%)$ & 0.82 \\
\hline Hypertension & $5(17.2 \%)$ & $3(14.3 \%)$ & 0.78 & $5(15.2 \%)$ & $3(17.6 \%)$ & 0.82 & $5(17.2 \%)$ & $3(14.3 \%)$ & 0.78 \\
\hline Diabetes mellitus & $0(0.0 \%)$ & $2(9.5 \%)$ & 0.090 & $0(0.0 \%)$ & $2(11.8 \%)$ & 0.044 & $0(0.0 \%)$ & $2(9.5 \%)$ & 0.090 \\
\hline Stroke & 0 & 0 & 1.00 & 0 & 0 & 1.00 & 0 & 0 & 1.00 \\
\hline Myocardial infarction & 0 & 0 & 1.00 & 0 & 0 & 1.00 & 0 & 0 & 1.00 \\
\hline Allergic rhinitis & $6(20.7 \%)$ & $4(19.0 \%)$ & 0.89 & $8(24.2 \%)$ & $2(11.8 \%)$ & 0.30 & $8(27.6 \%)$ & $2(9.5 \%)$ & 0.12 \\
\hline Polycystic ovarian syndrome & $1(3.4 \%)$ & $0(0.0 \%)$ & 0.39 & 0 & 0 & 1.00 & $0(0.0 \%)$ & $1(4.8 \%)$ & 0.24 \\
\hline
\end{tabular}

TABLE 4: Comparisons of biochemical profiles within 3 traditional Chinese medicine body constitutions (TCMBC).

\begin{tabular}{|c|c|c|c|c|c|c|c|c|c|}
\hline \multirow{2}{*}{ Parameters } & \multicolumn{3}{|c|}{ Yang deficiency (YangDC) } & \multicolumn{3}{|c|}{ Yin deficiency (YinDC) } & \multicolumn{3}{|c|}{ Phlegm-stasis (PSC) } \\
\hline & $(+) n=29$ & $(-) n=21$ & $P$ & (+) $n=33$ & $(-) n=17$ & $P$ & $(+) n=29$ & $(-) n=21$ & $P$ \\
\hline Fasting & $92.7(12.7)$ & $99.3(24.6)$ & 022 & $94.1(12.2)$ & $98.2(27.6)$ & 0.47 & $92.9(13.0)$ & $99.0(24.5)$ & 0.25 \\
\hline HbAlc & $6.0(0.4)$ & $6.0(0.7)$ & & $6.0(0.4)$ & $6.0(0.7)$ & 0.86 & $6.0(0.4)$ & $6.0(0.7)$ & 0.72 \\
\hline eAG & $126.4(11.7)$ & $126.0(19.2)$ & 0 & $125.9(11.6)$ & $126.8(20.8)$ & 0.85 & $126.9(11.6)$ & $125.4(19.3)$ & 0.74 \\
\hline HDL & $3.3(10.9)$ & $9.3)$ & 0.53 & $47.6(10.5)$ & & 0.24 & $46.8(9.9)$ & & 0.14 \\
\hline VLDL & $28.3(20.2)$ & 33.1 & 0.41 & $32.7(20.5)$ & & 0.22 & $30.4(20.3)$ & & 0.96 \\
\hline LDL & $129.9(35.0)$ & 131.1 & 0.91 & $130.6(36.4)$ & $130.0(34.2)$ & 0.95 & $130.0(36.1)$ & 131 & 0.93 \\
\hline Total & $199.8(41.5)$ & 203. & & $203.2(40.1)$ & 197 & 0.63 & $199.7(43.2)$ & $.2)$ & 0.76 \\
\hline lesterol/HDL & 4.3 & 9) & 0.70 & $4.4(1.1)$ & & 0.63 & 4.4( & $0)$ & 0.73 \\
\hline $\mathrm{LDL} / \mathrm{H}$ & $2.8(1.0)$ & $2.8(0.7)$ & 0.94 & $2.8(0.8)$ & $2.8(1.0)$ & 0.99 & $2.9(0.9)$ & $2.7(0.8)$ & 0.57 \\
\hline Triglyceride & $46.2(120.0)$ & $181.7(139.1)$ & 0.34 & $175.7(140.0)$ & $132.9(99.4)$ & 0.27 & $156.6(120.5)$ & $167.5(141.0)$ & 0.77 \\
\hline Leptin & $29.7(24.8)$ & $15.9(9.9)$ & 0.020 & $28.8(23.5)$ & $14.4(9.6)$ & 0.020 & $25.9(21.4)$ & $21.0(20.5)$ & 0.42 \\
\hline Insulin & $11.9(5.6)$ & $12.4(4.5)$ & 0.73 & $13.0(5.3)$ & $10.4(4.4)$ & 0.088 & $12.3(5.0)$ & $11.9(5.4)$ & 0.79 \\
\hline HOMA-IR & $2.8(1.7)$ & $3.6(3.0)$ & 0.21 & $3.1(1.6)$ & $3.3(3.4)$ & 0.82 & $2.9(1.6)$ & $3.5(3.1)$ & 0.35 \\
\hline Adiponectin & $7.4(5.0)$ & $7.6(4.4)$ & 0.90 & $7.6(5.1)$ & $7.2(4.0)$ & 0.78 & $7.4(5.2)$ & $7.6(4.0)$ & 0.87 \\
\hline
\end{tabular}

$\mathrm{HbAlc}$, glycated hemoglobin; eAG, estimated average glucose; HDL, high-density lipoprotein; VLDL, very low-density lipoprotein; LDL, low-density lipoprotein; HOMA-IR, homeostatic model assessment for insulin resistance.

deficiency patients had different hypertension [47]. The geolocation may cause the differences between these studies, since the proportion of hypertension and diabetes mellitus was both low in our study. Compared to Shang Hai, where the Li et al.' subjects' information was collected, Taiwan's latitude is lower, and the climate is warmer than Shang Hai. This fact may be the reason why the distribution of TCMBC was different from the previous study.
Moreover, we found the expression of genomic profile tended to be different among these three TCMBC. Genetic variations in the ADRB3, the $\beta_{3}$-adrenoceptor (i.e. Try64Arg variant), are also associated with obesity. ADRB3 stimulates the mobilization of lipids from the white fat cell and increases thermogenesis in the brown fat cell. Decreased function of ADRB3 in white adipose tissue could slow lipolysis and cause the retention of lipids in fat cells [48]. YangDC implies a diminishing energy level in the body's 
TABLE 5: Comparisons of genomic profiles within 3 traditional Chinese medicine body constitutions (TCMBC).

\begin{tabular}{|c|c|c|c|c|c|c|c|c|c|c|c|}
\hline \multirow{2}{*}{ Parameters } & & & \multicolumn{3}{|c|}{ Yang deficiency (YangDC) } & \multicolumn{3}{|c|}{ Yin deficiency (YinDC) } & \multicolumn{3}{|c|}{ Phlegm-stasis (PSC) } \\
\hline & & & (+) $n=29$ & $(-) n=21$ & $P$ & (+) $n=33$ & $(-) n=17$ & $P$ & $(+) n=29$ & $(-) n=21$ & $P$ \\
\hline \multicolumn{12}{|c|}{ Obesity polymorphisms } \\
\hline \multirow{6}{*}{ ADRB2 (starch) } & \multirow{3}{*}{ rs1042713 } & Low & $\begin{array}{c}11 \\
(37.9 \%)\end{array}$ & $5(23.8 \%)$ & \multirow[t]{2}{*}{0.50} & $\begin{array}{c}13 \\
(39.4 \%)\end{array}$ & $3(17.6 \%)$ & \multirow[t]{2}{*}{0.20} & $9(31.0 \%)$ & $7(33.3 \%)$ & \multirow[t]{2}{*}{0.79} \\
\hline & & Medium & $12(41.4 \%)$ & $12(57.1 \%)$ & & $\begin{array}{c}13 \\
(39.4 \%)\end{array}$ & $11(64.7 \%)$ & & $15(51.7 \%)$ & $9(42.9 \%)$ & \\
\hline & & High & $6(20.7 \%)$ & $4(19.0 \%)$ & & $7(21.2 \%)$ & $3(17.6 \%)$ & & $5(17.2 \%)$ & $5(23.8 \%)$ & \multirow{3}{*}{0.74} \\
\hline & \multirow{3}{*}{ rs1042714 } & Low & 27 (93.1\%) & $\begin{array}{c}19 \\
(90.5 \%)\end{array}$ & 0.74 & $31(93.9 \%)$ & $15(88.2 \%)$ & \multirow[t]{2}{*}{0.48} & $27(93.1 \%)$ & $\begin{array}{c}19 \\
(90.5 \%)\end{array}$ & \\
\hline & & Medium & $2(6.9 \%)$ & $2(9.5 \%)$ & & $2(6.1 \%)$ & $2(11.8 \%)$ & & $2(6.9 \%)$ & $2(9.5 \%)$ & \\
\hline & & Low & $1(3.4 \%)$ & $0(0.0 \%)$ & 0.091 & $1(3.0 \%)$ & $0(0.0 \%)$ & 0.15 & $1(3.4 \%)$ & $0(0.0 \%)$ & 0.63 \\
\hline \multirow{3}{*}{ ADRB3 (visceral) } & \multirow{3}{*}{ rs4994 } & Medium & $2(6.9 \%)$ & $6(28.6 \%)$ & & $3(9.1 \%)$ & $5(29.4 \%)$ & & $4(13.8 \%)$ & $4(19.0 \%)$ & \\
\hline & & High & $\begin{array}{c}26 \\
(89.7 \%)\end{array}$ & $15(71.4 \%)$ & & $\begin{array}{c}29 \\
(87.9 \%)\end{array}$ & $12(70.6 \%)$ & & $\begin{array}{c}24 \\
(82.8 \%)\end{array}$ & $17(81.0 \%)$ & \\
\hline & & Low & $3(10.3 \%)$ & $3(14.3 \%)$ & 0.70 & $3(9.1 \%)$ & $3(17.6 \%)$ & 0.46 & $2(6.9 \%)$ & $4(19.0 \%)$ & 0.42 \\
\hline \multirow{2}{*}{$\begin{array}{l}\text { GNB3 } \\
\text { (metabolism) }\end{array}$} & \multirow{2}{*}{ rs5443 } & Medium & $\begin{array}{c}13 \\
(44.8 \%)\end{array}$ & $\begin{array}{c}11 \\
(52.4 \%)\end{array}$ & & $\begin{array}{c}15 \\
(45.5 \%)\end{array}$ & $9(52.9 \%)$ & & $15(51.7 \%)$ & $9(42.9 \%)$ & \\
\hline & & High & $\begin{array}{c}13 \\
(44.8 \%) \\
\end{array}$ & $7(33.3 \%)$ & & $\begin{array}{c}15 \\
(45.5 \%)\end{array}$ & $5(29.4 \%)$ & & $12(41.4 \%)$ & $8(38.1 \%)$ & \\
\hline \multirow[t]{3}{*}{ UCP1 (stubborn) } & \multirow[t]{3}{*}{ rs1800592 } & Low & $3(10.3 \%)$ & $5(23.8 \%)$ & \multirow[t]{3}{*}{0.18} & $4(12.1 \%)$ & $4(23.5 \%)$ & \multirow[t]{3}{*}{0.45} & $5(17.2 \%)$ & $3(14.3 \%)$ & \multirow[t]{3}{*}{0.052} \\
\hline & & Medium & $\begin{array}{c}16 \\
(55.2 \%)\end{array}$ & $13(61.9 \%)$ & & $\begin{array}{c}19 \\
(57.6 \%)\end{array}$ & $10(58.8 \%)$ & & $\begin{array}{c}13 \\
(44.8 \%)\end{array}$ & $\begin{array}{c}16 \\
(76.2 \%)\end{array}$ & \\
\hline & & High & $\begin{array}{c}10 \\
(34.5 \%)\end{array}$ & $3(14.3 \%)$ & & $\begin{array}{c}10 \\
(30.3 \%)\end{array}$ & $3(17.6 \%)$ & & $\begin{array}{c}11 \\
(37.9 \%)\end{array}$ & $2(9.5 \%)$ & \\
\hline \multirow{3}{*}{ FTO (appetite) } & \multirow{3}{*}{ rs9939609 } & Low & $\begin{array}{c}20 \\
(69.0 \%)\end{array}$ & $15(71.4 \%)$ & \multirow[t]{3}{*}{0.46} & $\begin{array}{c}24 \\
(72.7 \%)\end{array}$ & $11(64.7 \%)$ & \multirow[t]{3}{*}{0.37} & $\begin{array}{c}20 \\
(69.0 \%)\end{array}$ & $15(71.4 \%)$ & \multirow[t]{3}{*}{0.46} \\
\hline & & Medium & $7(24.1 \%)$ & $6(28.6 \%)$ & & $7(21.2 \%)$ & $6(35.3 \%)$ & & $7(24.1 \%)$ & $6(28.6 \%)$ & \\
\hline & & High & $2(6.9 \%)$ & $0(0.0 \%)$ & & $2(6.1 \%)$ & $0(0.0 \%)$ & & $2(6.9 \%)$ & $0(0.0 \%)$ & \\
\hline
\end{tabular}

TABLE 6: Comparisons of demographic features, comorbidities, biochemical profiles, and genomic profiles within three-combined traditional Chinese medicine body constitutions (TCMBC) and balanced constitution (no inclined to any of three types) obesity enrollees.

\begin{tabular}{|c|c|c|c|}
\hline \multirow{2}{*}{ Parameters } & \multicolumn{3}{|c|}{ TCMBC } \\
\hline & Three-combined $(n=22)$ & Balanced $(n=11)$ & $P$ \\
\hline \multicolumn{4}{|l|}{ Demographic features } \\
\hline Gender & & & $<0.001$ \\
\hline Female & $20(90.9 \%)$ & $4(36.4 \%)$ & \\
\hline Male & $2(9.1 \%)$ & $7(63.6 \%)$ & \\
\hline Age (years), mean (SD) & $38.4(10.9)$ & $35.0(10.2)$ & 0.40 \\
\hline BMI, mean (SD) & $30.5(3.1)$ & $31.3(3.3)$ & 0.50 \\
\hline Waist-hip ratio (WHR), mean (SD) & $0.9(0.1)$ & $0.9(0.0)$ & 0.27 \\
\hline Waist-height ratio (WheR), mean (SD) & $0.6(0.1)$ & $0.6(0.0)$ & 0.47 \\
\hline Appetite & & & 0.53 \\
\hline Poor & $2(9.1 \%)$ & $0(0.0 \%)$ & \\
\hline Fair & $19(86.4 \%)$ & $10(90.9 \%)$ & \\
\hline Good & $1(4.5 \%)$ & $1(9.1 \%)$ & \\
\hline \multicolumn{4}{|l|}{ Food preference } \\
\hline Icy food & $7(31.8 \%)$ & $1(9.1 \%)$ & 0.15 \\
\hline Deep-fried food & $6(27.3 \%)$ & $0(0.0 \%)$ & 0.056 \\
\hline Smoking & $1(4.5 \%)$ & $0(0.0 \%)$ & 0.47 \\
\hline Alcohol consumption & $7(31.8 \%)$ & $7(63.6 \%)$ & 0.081 \\
\hline Exercise $\geq 30$ mins/week & $17(77.3 \%)$ & $8(72.7 \%)$ & 0.77 \\
\hline \multicolumn{4}{|l|}{ Comorbidities } \\
\hline Hyperlipidemia & $1(4.5 \%)$ & $0(0.0 \%)$ & 0.47 \\
\hline Hypertension & $4(18.2 \%)$ & $2(18.2 \%)$ & 1.00 \\
\hline Diabetes mellitus & $0(0.0 \%)$ & $2(18.2 \%)$ & 0.039 \\
\hline Stroke & 0 & 0 & 1.00 \\
\hline Myocardial infarction & 0 & 0 & 1.00 \\
\hline Allergic rhinitis & $6(27.3 \%)$ & $1(9.1 \%)$ & 0.23 \\
\hline Polycystic ovarian syndrome & 0 & 0 & 1.00 \\
\hline
\end{tabular}


TABLE 6: Continued.

\begin{tabular}{lccc}
\hline Parameters & & TCMBC \\
& Three-combined $(n=22)$ & Balanced $(n=11)$ & \\
\hline Biochemical profiles & & $101.7(33.2)$ & \\
Fasting sugar & $92.4(13.1)$ & $6.1(0.9)$ & $129.4(25.7)$ \\
HbAlc & $6.1(0.5)$ & $55.1(24.6)$ & 0.25 \\
eAG & $127.0(13.0)$ & $31.6(19.2)$ & 0.73 \\
HDL & $47.6(10.3)$ & $129.9(27.7)$ & 0.23 \\
VLDL & $31.5(21.9)$ & $201.6(29.3)$ & 0.99 \\
LDL & $126.9(34.1)$ & $4.4(1.0)$ & 0.80 \\
Total cholesterol & $199.2(42.8)$ & $2.8(0.7)$ \\
Total cholesterol/HDL & $4.3(1.2)$ & $165.1(110.7)$ & 0.87 \\
LDL/HDL & $2.8(0.9)$ & $11.3(8.8)$ & 0.92 \\
Triglyceride & $163.2(132.2)$ & $11.1(4.7)$ \\
Leptin & $27.7(23.9)$ & $3.9(4.0)$ \\
Insulin & $12.1(5.3)$ & $6.5(3.2)$ \\
HOMA-IR & $2.9(1.7)$ & 0.97 \\
Adiponectin & $7.2(5.2)$ & 0.036 \\
\hline
\end{tabular}

HbAlc, glycated hemoglobin; eAG, estimated average glucose; HDL, high-density lipoprotein; VLDL, very low-density lipoprotein; LDL, low-density lipoprotein; HOMA-IR, homeostatic model assessment for insulin resistance.

TABLE 7: The genomic profile difference among three-combined traditional Chinese medicine body constitutions (TCMBC) and balanced constitution (not inclined to any of three types) obesity enrollees.

\begin{tabular}{|c|c|c|c|c|c|}
\hline \multirow[t]{2}{*}{ Obesity polymorphisms } & \multicolumn{3}{|c|}{ TCMBC } & \multicolumn{2}{|r|}{$P$} \\
\hline & \multirow{3}{*}{ rs1042713 } & Low & $8(36.4 \%)$ & $2(18.2 \%)$ & 0.33 \\
\hline \multirow{4}{*}{ ADRB2 (starch) } & & Medium & $10(45.5 \%)$ & $8(72.7 \%)$ & \\
\hline & & High & $4(18.2 \%)$ & $1(9.1 \%)$ & \\
\hline & \multirow{2}{*}{ rs1042714 } & Low & $20(90.9 \%)$ & $9(81.8 \%)$ & 0.45 \\
\hline & & Medium & $2(9.1 \%)$ & $2(18.2 \%)$ & \\
\hline \multirow{3}{*}{ ADRB3 (visceral) } & \multirow{3}{*}{ rs4994 } & Low & $1(4.5 \%)$ & $0(0.0 \%)$ & 0.14 \\
\hline & & Medium & $1(4.5 \%)$ & $3(27.3 \%)$ & \\
\hline & & High & $20(90.9 \%)$ & $8(72.7 \%)$ & \\
\hline \multirow{3}{*}{ GNB3 (metabolism) } & \multirow{3}{*}{ rs5443 } & Low & $1(4.5 \%)$ & $2(18.2 \%)$ & 0.34 \\
\hline & & Medium & $11(50.0 \%)$ & $6(54.5 \%)$ & \\
\hline & & High & $10(45.5 \%)$ & $3(27.3 \%)$ & \\
\hline \multirow{3}{*}{ UCP1 (stubborn) } & \multirow{3}{*}{ rs1800592 } & Low & $3(13.6 \%)$ & $3(27.3 \%)$ & 0.28 \\
\hline & & Medium & $9(40.9 \%)$ & $6(54.5 \%)$ & \\
\hline & & High & $10(45.5 \%)$ & $2(18.2 \%)$ & \\
\hline \multirow{3}{*}{ FTO (appetite) } & \multirow{3}{*}{ rs9939609 } & Low & $16(72.7 \%)$ & $8(72.7 \%)$ & 0.53 \\
\hline & & Medium & $4(18.2 \%)$ & $3(27.3 \%)$ & \\
\hline & & High & $2(9.1 \%)$ & $0(0.0 \%)$ & \\
\hline
\end{tabular}

physiological functioning and can also easily cause metabolic waste accumulation and lead to obesity [44]. UCP1 plays a vital role in metabolic and energy balance and regulation, cold- and diet-induced thermogenesis, and decreasing the production of reactive oxygen species by mitochondria, which are mechanisms associated with the pathogenesis of obesity $[49,50]$. PSC refers to individuals' dynamic interaction between yin and yang that is decelerated and less efficient, and individuals may express some physical symptoms [21]. Once phlegm and stasis are formed, they would become fat accumulation and lead to obesity [44].

This study reported the relationship between TCMBC, leptin, and obesity-related SNPs. This study used biochemical parameters and genomic profiles to access TCMBC for bridging the gap between TCM theory and obesity polymorphisms. Despite its contributions, this study has several limitations. First, in our study, the patients were relatively young, and only one medical center was involved, and therefore, the generalizability may be concerned, especially the geolocation may be influential for determining TCMBC. Second, we used BCQ for assessing TCMBC, which is convenient to use and well-validated in Taiwan but has fewer constitution classifications than other studies about TCMBC [51-55]. Third, the BCQ used in this study assessed the presence or absence of three different TCMBC separately, but the three constitutions were not independent. Although it may reflect the complexity of obesity, the inference between combined TCMBC on parameters may influence the results. The constitution questionnaire to classify the subjects to a single TCMBC developed by professor Wang may be considered as well as BCQ in 
future studies about TCMBC classification among the obesity population [52]. Last, since this study design is cross-sectional, our study only suggested a possible association between TCMBC, leptin, and SNPs. The cause-causal relationships remain unclear, and further studies are still needed.

\section{Conclusions}

We found that the obese patients tended to have YinDC, while leptin is related to YangDC and YinDC, but not related to PSC. There is a trend towards near-statistical differences between the ADRB3 gene and YangDC, UCP1 gene, and PSC, and more research is needed to explain this relationship.

\section{Abbreviations}

$\begin{array}{ll}\text { ADRB2: } & \text { Adrenergic receptor beta-2 } \\ \text { ADRB3: } & \text { Adrenergic receptor beta-3 } \\ \text { BCQ: } & \text { Body constitution questionnaire } \\ \text { BMI: } & \text { Body mass index } \\ \text { eAG: } & \text { Estimated average glucose } \\ \text { FTO: } & \text { Fat mass- and obesity-associated } \\ \text { GNB3: } & \text { Guanine nucleotide-binding protein } \\ \text { HbA1c: } & \text { Hemoglobin A1C } \\ \text { HC: } & \text { Hip circumference } \\ \text { HDL-C: } & \text { High-density lipoprotein-cholesterol } \\ \text { HOMA- } & \text { Homeostatic model assessment for insulin } \\ \text { IR: } & \text { resistance } \\ \text { IRB: } & \text { Institutional Review Board } \\ \text { LDL-C: } & \text { Low-density lipoprotein-cholesterol } \\ \text { PSC: } & \text { Phlegm-stasis constitution } \\ \text { SNPs: } & \text { Single nucleotide polymorphisms } \\ \text { TCM: } & \text { Traditional Chinese medicine } \\ \text { TCMBC: } & \text { Traditional Chinese medicine body constitution } \\ \text { TG: } & \text { Triglyceride } \\ \text { UCP1: } & \text { Uncoupling protein 1 } \\ \text { VLDL: } & \text { Very low-density lipoprotein } \\ \text { WC: } & \text { Waist circumference } \\ \text { YangDC: } & \text { Yang deficiency constitution } \\ \text { YinDC: } & \text { Yin deficiency constitution. } \\ & \end{array}$

\section{Data Availability}

The data used to support the findings of this study are available from the corresponding author upon request.

\section{Conflicts of Interest}

The authors declare that they have no conflicts of interest.

\section{Authors' Contributions}

Hsing-Yu Chen devised the study's idea and analyzed the data, and Hsiang-I Hou prepared the manuscript. Jang-Jih $\mathrm{Lu}$ and Shih-Cheng Chang were responsible for genomic profile experiments and data interpretation. Hsueh-Yu Li commented on the study design and collected patients. KunHao Jiang and Jiun-Liang Chen provided TCM's viewpoint and revised the article. All authors approved the final version. Hsiang-I Hou and Hsing-Yu Chen equally contributed to this work.

\section{Acknowledgments}

The authors thank the department of laboratory medicine of the Chang-Gung Memorial Hospital for providing the blood and gene test technical support, which was supported by the funding from the Chang-Gung Memorial Foundation (CMRPG1H0051, CMRPG1H0052, CMRPG1F0011, CORPG1H0041, and CORPG1H0051). Also, the authors thank Dr. Pei-Ju Tsai for data collection at the initial stage of this study and Dr. Yi-Hsuan Lin for the result interpretation.

\section{References}

[1] F. B. Hu, "Obesity and mortality: watch your waist, not just your weight," Archives of Internal Medicine, vol. 167, no. 9, pp. 875-876, 2007.

[2] M. M. Finucane, G. A. Stevens, M. J. Cowan et al., "National, regional, and global trends in body-mass index since 1980: systematic analysis of health examination surveys and epidemiological studies with 960 country-years and 9.1 million participants," The Lancet, vol. 377, no. 9765, pp. 557-567, 2011.

[3] E. A. Finkelstein, O. A. Khavjou, H. Thompson et al., "Obesity and severe obesity forecasts through 2030," American Journal of Preventive Medicine, vol. 42, no. 6, pp. 563-570, 2012.

[4] L.-C. Hwang, C.-H. Bai, and C.-J. Chen, "Prevalence of obesity and metabolic syndrome in Taiwan," Journal of the Formosan Medical Association, vol. 105, no. 8, pp. 626-635, 2006.

[5] M. D. Jensen, D. H. Ryan, C. M. Apovian et al., "2013 AHA/ ACC/TOS guideline for the management of overweight and obesity in adults," Journal of the American College of Cardiology, vol. 63, no. 25, pp. 2985-3023, 2014.

[6] A. J. Stunkard, J. R. Harris, N. L. Pedersen, and G. E. McClearn, "The body-mass index of twins who have been reared apart," New England Journal of Medicine, vol. 322, no. 21, pp. 1483-1487, 1990

[7] H. H. M. Maes, M. C. Neale, and L. J. Eaves, "Genetic and environmental factors in relative body weight and human adiposity," Behavior Genetics, vol. 27, no. 4, pp. 325-351, 1997.

[8] M. O. Goodarzi, "Genetics of obesity: what genetic association studies have taught us about the biology of obesity and its complications," The Lancet Diabetes and Endocrinology, vol. 6, no. 3, pp. 223-236, 2018.

[9] T.-J. Hsiao and E. Lin, "Evaluation of the glutamine 27 glutamic acid polymorphism in the adrenoceptor $\beta_{2}$ surface gene on obesity and metabolic phenotypes in taiwan," Journal of Investigative Medicine, vol. 62, no. 2, pp. 310-315, 2014.

[10] M. Daghestani, M. Daghestani, M. Daghistani et al., “ADRB3 polymorphism rs4994 (Trp64Arg) associates significantly with bodyweight elevation and dyslipidaemias in Saudis but not rs1801253 (Arg389Gly) polymorphism in ARDB1," Lipids in Health and Disease, vol. 17, no. 1, p. 58, 2018.

[11] H.-L. Li, Y.-J. Zhang, X.-P. Chen, J.-Q. Luo, S.-Y. Liu, and Z.-L. Zhang, "Association between GNB3 c.825C > T polymorphism and the risk of overweight and obesity: a metaanalysis," Meta Gene, vol. 9, pp. 18-25, 2016.

[12] S. Chathoth, M. H. Ismail, C. Vatte et al., "Association of Uncoupling Protein 1 (UCP1) gene polymorphism with 
obesity: a case-control study," BMC Medical Genetics, vol. 19, no. 1, p. 203, 2018.

[13] R. Fredriksson, M. Hägglund, P. K. Olszewski et al., "The obesity gene, FTO, is of ancient origin, up-regulated during food deprivation and expressed in neurons of feeding-related nuclei of the brain," Endocrinology, vol. 149, no. 5, pp. 2062-2071, 2008.

[14] A. Scuteri, S. Sanna, W.-M. Chen et al., "Genome-wide association scan shows genetic variants in the FTO gene are associated with obesity-related traits," PLoS Genetics, vol. 3, no. 7, p. e115, 2007.

[15] H. You, T. Zhang, W. Feng, and Y. Gai, “Association of TCM body constitution with insulin resistance and risk of diabetes in impaired glucose regulation patients," BMC Complementary and Alternative Medicine, vol. 17, no. 1, p. 459, 2017.

[16] L. Li, H. Yao, J. Wang, Y. Li, and Q. Wang, "The role of Chinese medicine in health maintenance and disease prevention: application of constitution theory," American Journal of Chinese Medicine, vol. 47, no. 3, pp. 495-506, 2019.

[17] J.-S. Lin, L.-L. Chen, J.-D. Lin et al., "BCQ-: a body constitution questionnaire to assess Yin-Xu. part II: evaluation of reliability and validity," Forschende Komplementärmedizin/ Research in Complementary Medicine, vol. 19, no. 6, pp. 285-292, 2012.

[18] L.-L. Chen, J.-S. Lin, J.-D. Lin et al., "BCQ+: a body constitution questionnaire to assess Yang-Xu. part II: Evaluation of reliability and validity," Complementary Medicine Research, vol. 16, no. 1, pp. 20-27, 2009.

[19] Y.-C. Su, L.-L. Chen, J.-D. Lin, J.-S. Lin, Y.-c. Huang, and J.-S. Lai, "BCQ+: a body constitution questionnaire to assess Yang-Xu. part I: establishment of a first final version through a Delphi process," Complementary Medicine Research, vol. 15, no. 6, pp. 327-334, 2008.

[20] J.-D. Lin, L.-L. Chen, J.-S. Lin, C.-H. Chang, Y.-C. Huang, and Y.-C. Su, "BCQ-: a body constitution questionnaire to assess Yin-Xu. part I: establishment of a provisional version through a Delphi process," Forschende Komplementärmedizin/Research in Complementary Medicine, vol. 19, no. 5, pp. 234-241, 2012.

[21] J.-D. Lin, J.-S. Lin, L.-L. Chen, C.-H. Chang, Y.-C. Huang, and Y.-C. Su, "BCQs: a body constitution questionnaire to assess stasis in traditional Chinese medicine," European Journal of Integrative Medicine, vol. 4, no. 4, pp. e379-e391, 2012.

[22] Y.-C. Chou, C.-N. Tsai, Y.-S. Lee, and J.-S. Pei, “Association of adrenergic receptor gene polymorphisms with adolescent obesity in Taiwan," Pediatrics International, vol. 54, no. 1, pp. 111-116, 2012.

[23] V. Large, L. Hellström, S. Reynisdottir et al., "Human beta-2 adrenoceptor gene polymorphisms are highly frequent in obesity and associate with altered adipocyte beta- 2 adrenoceptor function," Journal of Clinical Investigation, vol. 100, no. 12, pp. 3005-3013, 1997.

[24] J. A. Martínez, M. S. Corbalán, A. Sánchez-Villegas, L. Forga, A. Marti, and M. A. Martínez-González, "Obesity risk is associated with carbohydrate intake in women carrying the Gln27Glu beta2-adrenoceptor polymorphism," The Journal of Nutrition, vol. 133, no. 8, pp. 2549-2554, 2003.

[25] P.-J. Tsai, S.-C. Ho, L.-P. Tsai et al., "Lack of relationship between $\beta_{3}$-adrenergic receptor gene polymorphism and gestational diabetes mellitus in a Taiwanese population," Metabolism, vol. 53, no. 9, pp. 1136-1139, 2004.

[26] J. A. Ryuk, X. Zhang, B.-S. Ko, J. W. Daily, and S. Park, "Association of $\beta_{3}$-adrenergic receptor rs4994 polymorphisms with the risk of type 2 diabetes: a systematic review and meta- analysis," Diabetes Research and Clinical Practice, vol. 129, pp. 86-96, 2017.

[27] H. Kim-Motoyama, K. Yasuda, T. Yamaguchi et al., "A mutation of the $\beta_{3}$-adrenergic receptor is associated with visceral obesity but decreased serum triglyceride," Diabetologia, vol. 40, no. 4, pp. 469-472, 1997.

[28] K. D. Ko, K. K. Kim, H. S. Suh, and I. C. Hwang, "Associations between the GNB3 C825T polymorphism and obesity-related metabolic risk factors in Korean obese women," Journal of Endocrinological Investigation, vol. 37, no. 11, pp. 1117-1120, 2014.

[29] T.-J. Hsiao, Y. Hwang, C.-H. Liu, H.-M. Chang, and E. Lin, "Association of the C825T polymorphism in the GNB3 gene with obesity and metabolic phenotypes in a Taiwanese population," Genes and Nutrition, vol. 8, no. 1, pp. 137-144, 2013.

[30] R. A. Busiello, S. Savarese, and A. Lombardi, "Mitochondrial uncoupling proteins and energy metabolism," Frontiers in Physiology, vol. 6, p. 36, 2015.

[31] K. Nakayama, H. Miyashita, Y. Yanagisawa, and S. Iwamoto, "Seasonal effects of UCP1 gene polymorphism on visceral fat accumulation in Japanese adults," PLoS One, vol. 8, no. 9, Article ID e74720, 2013.

[32] M. Dhall, M. M. Chaturvedi, U. Rai, and S. Kapoor, "Sexdependent effects of the UCP1 -3826 A/G polymorphism on obesity and blood pressure," Ethnicity and Disease, vol. 22, no. 2, pp. 181-184, 2012.

[33] M. Claussnitzer, S. N. Dankel, K.-H. Kim et al., "FTO obesity variant circuitry and adipocyte browning in humans," New England Journal of Medicine, vol. 373, no. 10, pp. 895-907, 2015.

[34] T.-J. Hsiao and E. Lin, "Association of a common rs9939609 variant in the fat mass and obesity-associated (FTO) gene with obesity and metabolic phenotypes in a Taiwanese population: a replication study," Journal of Genetics, vol. 95, no. 3, pp. 595-601, 2016.

[35] E. Karra, O. G. O’Daly, A. I. Choudhury et al., "A link between FTO, ghrelin, and impaired brain food-cue responsivity," Journal of Clinical Investigation, vol. 123, no. 8, pp. 3539-3551, 2013.

[36] C. N. Ayina, J. J. Noubiap, L. S. Etoundi Ngoa et al., "Association of serum leptin and adiponectin with anthropomorphic indices of obesity, blood lipids and insulin resistance in a Sub-Saharan African population," Lipids in Health and Disease, vol. 15, p. 96, 2016.

[37] A. Lubkowska, A. Radecka, I. Bryczkowska, I. Rotter, M. Laszczyńska, and W. Dudzińska, "Serum adiponectin and leptin concentrations in relation to body fat distribution, hematological indices and lipid profile in humans," International Journal of Environmental Research and Public Health, vol. 12, no. 9, pp. 11528-11548, 2015.

[38] J. L. Chan, K. Heist, A. M. DePaoli, J. D. Veldhuis, and C. S. Mantzoros, "The role of falling leptin levels in the neuroendocrine and metabolic adaptation to short-term starvation in healthy men," Journal of Clinical Investigation, vol. 111, no. 9, pp. 1409-1421, 2003.

[39] F. Lönnqvist, P. Arner, L. Nordfors, and M. Schalling, "Overexpression of the obese (ob) gene in adipose tissue of human obese subjects," Nature Medicine, vol. 1, no. 9, pp. 950-953, 1995.

[40] K. Diaoyuan and S. Yongxia, "A brief history of human constitutionology," Journal of Traditional Chinese Medicine=Chung I Tsa Chih Ying Wen pan, vol. 26, no. 3, pp. 230-238, 2006. 
[41] D. M. Duan, J. Y. Jhang, S. Wu, M. S. Teng, L. A. Hsu, and Y. L. Ko, "Modification effect of sex and obesity on the correlation of LEP polymorphisms with leptin levels in Taiwanese obese women," Molecular Genetics and Genomic Medicine, vol. 8, no. 3, Article ID e1113, 2020.

[42] W.-C. Li, K.-Y. Hsiao, I.-C. Chen, Y.-C. Chang, S.-H. Wang, and $\mathrm{K} . \mathrm{H}$. Wu, "Serum leptin is associated with cardiometabolic risk and predicts metabolic syndrome in Taiwanese adults," Cardiovascular Diabetology, vol. 10, no. 1, p. 36, 2011.

[43] J. Li, "Clinical experience in acupuncture treatment of obesity," Journal of Traditional Chinese Medicine, vol. 19, no. 1, pp. 48-51, 1999.

[44] M. Li, S. Mo, Y. Lv, Z. Tang, and J. Dong, "A study of traditional Chinese medicine body constitution associated with overweight, obesity, and underweight," Evidence-Based Complementary and Alternative Medicine, vol. 2017, Article ID 7361896, 7 pages, 2017.

[45] S. J. Grant, R. N. Schnyer, D. H. Chang, P. Fahey, and A. Bensoussan, "Interrater reliability of Chinese medicine diagnosis in people with pre-diabetes," Evidence-Based Complementary and Alternative Medicine, vol. 2013, Article ID 710892, , 2013.

[46] Y. Zhu, H. Shi, Q. Wang et al., “Association between nine types of TCM constitution and five chronic diseases: a correspondence analysis based on a sample of 2,660 participants," Evidence-Based Complementary and Alternative Medicine, vol. 2017, Article ID 9439682, , 2017.

[47] Y. Li, X. H. Li, X. Huang et al., "Individualized prevention against hypertension based on Traditional Chinese Medicine Constitution Theory: a large community-based retrospective, STROBE-compliant study among Chinese population," Medicine (Baltimore), vol. 96, no. 46, Article ID e8513, 2017.

[48] K. Masuo, "Roles of beta2- and beta3-adrenoceptor polymorphisms in hypertension and metabolic syndrome," International Journal of Hypertension, vol. 2010, Article ID 832821, 12 pages, 2010.

[49] V. Azzu and M. D. Brand, "The on-off switches of the mitochondrial uncoupling proteins," Trends in Biochemical Sciences, vol. 35, no. 5, pp. 298-307, 2010.

[50] L. T. Dalgaard and O. Pedersen, "Uncoupling proteins: functional characteristics and role in the pathogenesis of obesity and Type II diabetes," Diabetologia, vol. 44 , no. 8 , pp. 946-965, 2001.

[51] Q. Wang, X.-j. Ren, S.-l. Yao, and H.-d. Wu, "Clinical observation on the endocrinal and immune functions in subjects with yin-deficiency constitution," Chinese Journal of Integrative Medicine, vol. 16, no. 1, pp. 28-32, 2010.

[52] J. Wang, Y. Li, C. Ni, H. Zhang, L. Li, and Q. Wang, "Cognition research and constitutional classification in Chinese medicine," The American Journal of Chinese Medicine, vol. 39, no. 04, pp. 651-660, 2011.

[53] Q. Wang, "Individualized medicine, health medicine, and constitutional theory in Chinese medicine," Frontiers of Medicine, vol. 6, no. 1, pp. 1-7, 2012.

[54] S. Yao and Q. Wang, "Molecular basis for cold-intolerant yang-deficient constitution of traditional Chinese medicine," The American Journal of Chinese Medicine, vol. 36, no. 05, pp. 827-834, 2008.

[55] J. Wang, Q. Wang, L. Li et al., "Phlegm-dampness constitution: genomics, susceptibility, adjustment and treatment with traditional Chinese medicine," The American Journal of Chinese Medicine, vol. 41, no. 02, pp. 253-262, 2013. 